\title{
Epilepsy and noncompaction
}

\author{
J. Finsterer • S. Zarrouk-Mahjoub
}

Published online: 21 November 2013

(C) The Author(s) 2013. This article is published with open access at Springerlink.com

Keywords Non-compaction · Left ventricular hypertrabeculation $\cdot$ Cardiomyopathy $\cdot$ Syncope . Arrhythmia $\cdot$ Epilepsy $\cdot$ Seizures

\section{Dear Sir,}

With interest we read the article by Dello et al. about a patient with left ventricular hypertrabeculation/noncompaction (LVHT) and syncope being attributed to ventricular tachycardia [1]. We have the following comments and concerns.

Work-up for syncope not only requires cardiological but also neurological investigations. It is important not only to rule out epilepsy but also ischaemic stroke or cerebral bleeding. In this respect it is important to carry out multimodal cerebral MRI to receive not only parenchymatous but also vascular information. Stroke is particularly prevalent in LVHT patients because of embolism from thrombus formation within the intertrabecular spaces [2]. Single patients with LVHT may also develop atrial fibrillation, the most common risk factor for embolic stroke. A number of patients with LVHT also develop systolic dysfunction, another risk factor for stroke or embolism [3]. Did the described patient undergo cerebral MRI and what was the result? Where any old ischaemic lesions seen on TIRM sequences?

Syncope may also derive from stenosis of the extra-cranial cerebral arteries. Did the patient undergo carotid ultrasound or contrast-enhanced MR angiography to rule out high-grade stenosis of the internal carotid arteries or dissection of the carotid or vertebral arteries?

J. Finsterer ( $\square)$

Krankenanstalt Rudolfstiftung, Postfach 20, 1180 Vienna, Austria

e-mail: fifigs1@yahoo.de

S. Zarrouk-Mahjoub

Laboratory of Biochemistry, UR "Human Nutrition and Metabolic

Disorders" Faculty of Medicine Monastir, Monastir, Tunisie
A further neurological cause of syncope may be affection of the peripheral nerves [4]. Were there any indications for vegetative polyneuropathy in the described patient? Did he present with weakness, wasting, reduced tendon reflexes, or sensory disturbances? Was the patient seen by a neurologist to rule out or confirm acute or chronic polyneuropathy?

A fourth reason why the neurologist should see LVHT patients is the frequent association of LVHT with neuromuscular disorders [5]. Particularly muscle disease is highly prevalent among LVHT patients, although a causative relation between neuromuscular disorders and LVHT has not been proven yet.

It would be also interesting to receive a more detailed description of the paroxysmal activity on EEG? How often did the patient experience seizures? What type of seizures did he experience? Only focal or also generalised seizures? Did he also present with non-convulsive seizures? When was the onset of epilepsy, what was the seizure frequency, what were the most frequent triggers for seizures in this particular patient? Did he have a family history positive for epilepsy, did he have a history of birth trauma, cerebral hypoxia, fever cramps, traumatic brain injury, meningitis, or a history of neurosurgery? What was the cause of epilepsy and did he ever take antiepileptic drugs other than levetiracetam? How do the authors know that paroxysmal activity on EEG was secondary to arrhythmias and not primary due to a double trouble? It is not unusual that EEG is normal after a seizure, particularly after initiation of antiepileptic treatment. EEGs may be also abnormal in patients without epilepsy, such as in patients with migraine or other types of headache. Did the patient report a history of headache? Did he experience further seizures after discharge? How frequently did he attend the follow-ups? How were the follow-up EEGs? Was the family history positive for epilepsy?

Overall, there is a strong need to investigate LVHT patients with a history of syncope or epilepsy more 
thoroughly, particularly by referral to the neurologist. The neurologist must rule out stroke, epilepsy, carotid artery stenosis, polyneuropathy, or myopathy. Seizures in LVHT patients may not only be due to ischaemia during ventricular arrhythmias but also an independent second trouble in these patients.

Disclosures None

Open Access This article is distributed under the terms of the Creative Commons Attribution License which permits any use, distribution, and reproduction in any medium, provided the original author(s) and the source are credited.

\section{References}

1. Dello SA, Kievit C, Dunselman PH, Alings M. Loss of consciousness and convulsion induced by a ventricular tachycardia mimicking epilepsy in a patient with noncompaction cardiomyopathy: a case report. Neth Heart J 2013

2. Bhatia NL, Tajik AJ, Wilansky S, Steidley DE, Mookadam F. Isolated noncompaction of the left ventricular myocardium in adults: a systematic overview. J Card Fail. 2011;17:771-8.

3. Hutchins IM, Schaefer S. Progressive left ventricular noncompaction and systolic dysfunction. Exp Clin Cardiol. 2012;17:81-3.

4. Tramontozzi 3rd LA, Russell JA. Orthostatic intolerance in multifocal acquired demyelinating sensory and motor neuropathy. J Clin Neuromuscul Dis. 2012;14:34-9.

5. Finsterer J, Stöllberger C, Fazio G. Neuromuscular disorders in left ventricular hypertrabeculation/noncompaction. Curr Pharm Des. 2010;16:2895-904. 